\title{
Helicity wave functions for massless and massive spin-2 particles
}

\author{
D. Spehler \\ Instituto de Física Teórica, Universidade Estadual Paulista, Rua Pamplona 145, 01405 São Paulo, Brazil \\ and Université Louis Pasteur, Institut Universitaire de Technologie, 3 rue St. Paul, 67300 Strasbourg, France \\ S.F. Novaes \\ Instituto de Física Teórica, Universidade Estadual Paulista, Rua Pamplona 145, 01405 São Paulo, Brazil
}

(Received 24 June 1991)

\begin{abstract}
Starting from general properties of a spin-2 field, we construct helicity wave functions in the framework of the Weyl-van der Waerden spinor formalism. We discuss here the cases of massless and massive spin-2 particles.
\end{abstract}

A very efficient technique for evaluating multiparticle amplitudes is achieved by means of the helicity method. It turns out to be much more simple to calculate helicity matrix elements rather than evaluating the unpolarized amplitudes by summing the squared invariant amplitude over all possible spin states [1].

This method was improved [2,3] with the use of the Weyl-van der Waerden $\mathrm{SL}(2, C)$ spinors conjointly with the spinor calculus [4]. This way of dealing with helicity amplitudes is capable of eliminating the lengthy $\gamma$-matrices algebra by unifying the descriptions of Dirac spinors and Minkowski four-vectors. With this treatment it was feasible to obtain exact expressions for amplitudes containing a large number of external legs [5].

In the present work, we apply the Weyl-van der Waerden spinor method to spin-2 bosons. Making use of general properties of spin-2 helicity states, we construct helicity wave functions in spinorial form for massless and massive particles. Our results have a smooth interface to the method of Weyl-van der Waerden spinors applied for spin- $\frac{1}{2}$ and spin-1 particles [3] and also for spin- $\frac{3}{2}$ fermions [6].

A spin-2 field is described by a symmetric tensor $\Phi^{\mu \nu}$ which satisfies the Klein-Gordon equation. The tensor $\Phi^{\mu \nu}$ is traceless $\left(\Phi_{\mu}^{\mu}=0\right)$ and has null four-divergence $\left(\partial_{\mu} \Phi^{\mu \nu}=0\right)$. The solution of the Klein-Gordon equation for the spin-2 field of momentum $p$, in the absence of a source, is given by the plane-wave expansion

$$
\Phi^{\mu \nu}(x)=\int d^{3} p \sum_{\lambda}\left[\mathcal{E}^{\mu \nu}(p, \lambda) \exp \left(+i p_{\mu} x^{\mu}\right)+\text { H.c. }\right],
$$

where $\mathcal{E}^{\mu \nu}(p, \lambda)$ is the polarization tensor corresponding to states of definite helicities $(\lambda)$ which should satisfy

$$
\begin{aligned}
& \mathcal{E}^{\mu \nu}(p, \lambda)=\mathcal{E}^{\nu \mu}(p, \lambda), \\
& p_{\mu} \mathcal{E}^{\mu \nu}(p, \lambda)=p_{\nu} \mathcal{E}^{\mu \nu}(p, \lambda)=0, \\
& \mathcal{E}^{\mu}{ }_{\mu}(p, \lambda)=0 .
\end{aligned}
$$

The following normalization condition for the tensor $\mathcal{E}^{\mu \nu}$ can be adopted:

$$
\left[\mathcal{E}^{\mu \nu}(k, \lambda)\right]^{\dagger} \mathcal{E}_{\mu \nu}\left(k, \lambda^{\prime}\right)=\delta_{\lambda, \lambda^{\prime}}
$$

In order to apply the Weyl-van der Waerden method to spin-2 particles, we briefly summarize some results on spinor formalism. We use the conventions of Wess and Bagger [7], with $\operatorname{diag}\left(g_{\mu \nu}\right)=(1,-1,-1,-1)$, vector indices are denoted by Greek letters $(\mu, \nu, \ldots=0,1,2,3)$ and spinor ones are denoted by Latin letters $(a, b, \ldots=$ $1,2)$. We define the inner product between two spinors as

$$
\langle p q\rangle \equiv \epsilon^{a b} p_{b} q_{a}=p^{a} q_{a}, \quad\langle p q\rangle^{\dagger} \equiv \epsilon^{\dot{a} \dot{b}^{b}} \bar{p}_{\dot{b}} \bar{q}_{\dot{a}}=\bar{p}^{\dot{a}} \bar{q}_{\dot{a}}
$$

where $\epsilon^{a b}$ is the metric spinor

$$
\epsilon^{a b}=\left(\begin{array}{rr}
0 & 1 \\
-1 & 0
\end{array}\right)
$$

Any four-vector $p^{\mu}=\left(p^{0}, p^{i}\right)$ can be expressed as

$$
p^{\mu}=\frac{1}{2} \bar{\sigma}^{\mu \dot{a} b} P_{b \dot{a}},
$$

where $P_{a \dot{b}}=\sigma_{a b}^{\mu} p_{\mu}$. Therefore, the Lorentz invariant $p^{\mu} p_{\mu}$ can be written as

$$
p^{\mu} p_{\mu}=\frac{1}{2} P_{b \dot{a}} \bar{P}^{\dot{a} b} \equiv \frac{1}{2}\{P, \bar{P}\} .
$$

We start by analyzing massless spin- 2 particles. In this case, we require the invariance under the gauge transformation

$$
\Phi^{\mu \nu} \rightarrow \Phi^{\mu \nu}-\partial^{\mu} \Lambda^{\nu}-\partial^{\nu} \Lambda^{\mu}
$$

where $\Lambda^{\mu}$ are four arbitrary functions. This gauge transformation is able to decouple the vector and scalar parts of the tensor field [8]. We end up with only two physical helicity states, i.e., $\lambda= \pm 2$, which satisfy

$$
\left[\mathcal{E}^{\mu \nu}(p, \pm 2)\right]^{\dagger}=\mathcal{E}^{\mu \nu}(p, \mp 2) \text {. }
$$


Using Eq. (5) for each vectorial index of $\mathcal{E}^{\mu \nu}$, we can write the helicity tensor as

$$
\begin{aligned}
& \mathcal{E}^{\mu \nu}(p,+2)=\left(\frac{1}{2} \bar{\sigma}^{\mu \dot{a} b} E_{b \dot{a}}\right)\left(\frac{1}{2} \bar{\sigma}^{\nu \dot{c} d} F_{d \dot{c}}\right), \\
& \mathcal{E}^{\mu \nu}(p,-2)=\left(\frac{1}{2} \bar{\sigma}^{\mu \dot{a} b} G_{b \dot{a}}\right)\left(\frac{1}{2} \bar{\sigma}^{\nu \dot{c} d} H_{d \dot{c}}\right),
\end{aligned}
$$

where $E, F, G$, and $H$ are arbitrary spinors to be determined. Equations (1a)-(1c), (2), and (8) impose some constraints on these spinors. For instance, the conditions (1a) $-(1 c)$ require

$$
\begin{aligned}
& E^{a \dot{b}} F^{c \dot{d}}=E^{c \dot{d}} F^{a \dot{b}}, \\
& P^{a \dot{b}} E_{a \dot{b}} F_{c \dot{d}}=P^{a \dot{b}} G_{a \dot{b}} H_{c \dot{d}}=0, \\
& E_{a \dot{b}} P^{c \dot{d}} F_{c \dot{d}}=G_{a \dot{b}} P^{c \dot{d}} H_{c \dot{d}}=0, \\
& E_{a \dot{b}} F^{a \dot{b}}=G_{a \dot{b}} H^{a \dot{b}}=0,
\end{aligned}
$$

whereas the normalization and Hermiticity conditions demand

$$
\begin{aligned}
& E_{a \dot{b}} G^{a \dot{b}} F_{c \dot{d}} H^{c \dot{d}}=4 \\
& \left(E^{a \dot{b}}\right)^{\dagger}\left(F^{c \dot{d}}\right)^{\dagger}=G^{b \dot{a}} H^{d \dot{c}}
\end{aligned}
$$

We make use of the fact that, for a massless particle, the spinor $P_{a b}$ associated with the particle momentum acquires a simple form. Since $p^{2}=\frac{1}{2}\{P, \bar{P}\}=0, P_{a \dot{b}}$ should be written as the product of two momentum spinors: i.e.,

$$
P_{a \dot{b}}=p_{a} \bar{p}_{\dot{b}} .
$$

We search for an explicit form of $E, F, G$, and $H$ writing

$$
\begin{aligned}
& E_{a \dot{b}}=e_{a} \bar{e}_{\dot{b}}^{\prime}, \quad F_{a \dot{b}}=f_{a} \bar{f}_{\dot{b}}^{\prime}, \\
& G_{a \dot{b}}=g_{a} \bar{g}_{\dot{b}}^{\prime}, \quad H_{a \dot{b}}=h_{a} \bar{h}_{\dot{b}}^{\prime},
\end{aligned}
$$

where $g, g^{\prime}, \ldots, h, h^{\prime}$ are arbitrary spinors. The relation (10e) constraints $e=g^{\prime}, g=e^{\prime}, f=h^{\prime}$, and $h=f^{\prime}$. The relation (10b), taking into account Eq.(11), yields four possibilities: $e=p$ and $f=p ; e^{\prime}=p$ and $f^{\prime}=p$; $e=p$ and $f^{\prime}=p$; or $e^{\prime}=p$ and $f=p$. Nevertheless, Eq. (10a) makes only the first two possibilities acceptable with the additional constraint: $e^{\prime}=f^{\prime}$ in the former case and $e=f$ in the later one. In order to guarantee that $\mathcal{E}^{\mu \nu}(p,+2)$ corresponds to a positive-helicity state, the only possible choice is $e^{\prime}=f^{\prime}=p$ and $e=f$, i.e.,

$$
\begin{aligned}
& \mathcal{E}^{\mu \nu}(p,+2)=\frac{1}{2} \bar{\sigma}^{\mu \dot{a} b} \bar{\sigma}^{\nu \dot{c} d} \bar{p}_{\dot{a}} e_{b} \bar{p}_{\dot{c}} e_{d}, \\
& \mathcal{E}^{\mu \nu}(p,-2)=\frac{1}{2} \bar{\sigma}^{\mu \dot{a} b} \bar{\sigma}^{\nu \dot{c} d} \bar{e}_{\dot{a}} p_{b} \bar{e}_{\dot{c}} p_{d} .
\end{aligned}
$$

We can verify that $\mathcal{E}^{\mu \nu}(p,+2)$ corresponds indeed to a massless spin-2 particle with positive helicity [9]. In order to do this, let us assume that the particle is traveling in the $z$ direction, i.e., $p^{\mu}=(E, 0,0, E)$, where $E$ is its energy. In this case the momentum spinor is

$$
\bar{p}_{\dot{a}}=\sqrt{2 E}\left(\begin{array}{l}
0 \\
1
\end{array}\right)
$$

and the helicity tensor can be written as

$$
\begin{aligned}
\mathcal{E}^{\mu \nu}(p,+2) & \equiv \frac{1}{4} \bar{\sigma}^{\mu \dot{a} b} \bar{\sigma}^{\nu \dot{c} d} \mathcal{E}_{b \dot{a} d \dot{c}} \\
& =\frac{E}{2}\left(\begin{array}{cccc}
e_{2} e_{2} & -e_{2} e_{1} & -i e_{2} e_{1} & e_{2} e_{2} \\
-e_{1} e_{2} & e_{1} e_{1} & i e_{1} e_{1} & -e_{1} e_{2} \\
-i e_{1} e_{2} & i e_{1} e_{1} & -e_{1} e_{1} & -i e_{1} e_{2} \\
e_{2} e_{2} & -e_{2} e_{1} & -i e_{2} e_{1} & e_{2} e_{2}
\end{array}\right)
\end{aligned}
$$

Using the gauge freedom Eq. (7) in the momentum space, i.e., $\mathcal{E}^{\mu \nu} \rightarrow \mathcal{E}^{\mu \nu}-i\left(p^{\mu} \Lambda^{\nu}+p^{\nu} \Lambda^{\mu}\right)$ we can choose $\Lambda^{\mu}$ such that

$$
\Lambda^{1}=i \frac{e_{1} e_{2}}{2}, \quad \Lambda^{2}=-\frac{e_{1} e_{2}}{2}, \quad \Lambda^{0}=\Lambda^{3}=-i \frac{e_{2} e_{2}}{4} ;
$$

with this choice we have

$$
\mathcal{E}^{\mu \nu}(p,+2)=\frac{E}{2} e_{1} e_{1}\left(\begin{array}{rrrr}
0 & 0 & 0 & 0 \\
0 & 1 & i & 0 \\
0 & i & -1 & 0 \\
0 & 0 & 0 & 0
\end{array}\right)
$$

We can see that if we perform a rotation about the $z$ direction, i.e., $\mathcal{E}^{\prime \mu \nu}=R_{\alpha}^{\mu}(\theta, 0) R^{\nu}{ }_{\beta}(\theta, 0) \mathcal{E}^{\alpha \beta}$ we obtain $\mathcal{E}^{\prime \mu \nu}=\exp (+2 i \theta) \mathcal{E}^{\mu \nu}$ which shows that $\mathcal{E}^{\mu \nu}(p,+2)$ is a helicity +2 wave function.

From Eq. (10d) we obtain the normalization condition $|\langle p e\rangle|^{4}=4$, and consequently $\langle p e\rangle=\sqrt{2} \exp (i \omega)$, where $\omega$ is an arbitrary phase. If we define the spinor $r$ by

$$
e_{a}=\exp (i \omega) \sqrt{2} \frac{r_{a}}{\langle p r\rangle}
$$

we can write the final result for the helicity states of a massless spin-2 particle as

$$
\begin{aligned}
& \mathcal{E}^{\mu \nu}(p,+2)=\frac{1}{2} \bar{\sigma}^{\mu \dot{a} b} \bar{\sigma}^{\nu \dot{c} d} \exp (2 i \omega) \frac{\bar{p}_{\dot{a}} r_{b}}{\langle p r\rangle} \frac{\bar{p}_{\dot{c}} r_{d}}{\langle p r\rangle} \\
& \mathcal{E}^{\mu \nu}(p,-2)=\frac{1}{2} \bar{\sigma}^{\mu \dot{a} b} \bar{\sigma}^{\nu \dot{c} d} \exp (-2 i \omega) \frac{\bar{r}_{\dot{a}} p_{b}}{\langle p r\rangle^{\dagger}} \frac{\bar{r}_{\dot{c}} p_{d}}{\langle p r\rangle^{\dagger}}
\end{aligned}
$$

We see that $r$ is a gauge spinor. For instance, if we make two different choices of the spinor $r\left(r\right.$ and $\left.r^{\prime}\right)$ we obtain

$$
\mathcal{E}_{b \dot{a} d \dot{c}}\left(r^{\prime}\right)=\mathcal{E}_{b \dot{a} d \dot{c}}(r)-i\left(P_{d \dot{c}} \Lambda_{b \dot{a}}+P_{b \dot{a}} \Lambda_{d \dot{c}}\right),
$$

with

$$
\begin{aligned}
\Lambda_{b \dot{a}}= & \frac{2 i\left\langle r^{\prime} r\right\rangle}{\left\langle p r^{\prime}\right\rangle^{2}\langle p r\rangle^{2}} \\
& \times\left(\left\langle p r^{\prime}\right\rangle r_{b} \bar{p}_{\dot{a}}+\frac{1}{2}\left\langle r^{\prime} r\right\rangle p_{b} \bar{p}_{\dot{a}}\right) .
\end{aligned}
$$

If we take into account the results of Ref. [3] for the helicity of a massless spin-1 particle $\left[\varepsilon^{\mu}( \pm 1)\right]$ in spinorial form, we notice that the helicity states $\mathrm{Eq}$. (15) can be written as $\mathcal{E}^{\mu \nu}(p, \pm 2)=\varepsilon^{\mu}(p, \pm 1) \varepsilon^{\nu}(p, \pm 1)$. Equation (15), with $\omega=0$, agrees with Ref. [10] where the graviton helicity states are considered.

We can write the sum over the helicities as 


$$
\begin{aligned}
\sum_{\lambda= \pm 2} \mathcal{E}_{a \dot{b} c \dot{d}}(p, \lambda) & {\left[\mathcal{E}_{e \dot{f} g \dot{h}}(p, \lambda)\right]^{\dagger} } \\
& =\frac{4}{|\langle p r\rangle|^{4}}\left[r_{a} \bar{p}_{\dot{b}} r_{c} \bar{p}_{\dot{d}} \bar{r}_{\dot{e}} p_{f} \bar{r}_{\dot{g}} p_{h}+(p \leftrightarrow r)\right]
\end{aligned}
$$

which is equivalent to the vectorial form

$$
\begin{aligned}
& \sum_{\lambda= \pm 2} \mathcal{E}^{\mu \nu}(p, \lambda)\left[\mathcal{E}^{\alpha \beta}(p, \lambda)\right]^{\dagger} \\
&=\frac{1}{2}\left(\mathcal{P}^{\mu \alpha} \mathcal{P}^{\nu \beta}+\mathcal{P}^{\mu \beta} \mathcal{P}^{\nu \alpha}-\mathcal{P}^{\mu \nu} \mathcal{P}^{\alpha \beta}\right)
\end{aligned}
$$

where

$$
\mathcal{P}^{\mu \nu}=-g^{\mu \nu}+\frac{p^{\mu} r^{\nu}+p^{\nu} r^{\mu}}{p \cdot r} .
$$

We can see that the sum over the polarizations depends on the gauge spinor $r$ and that only the physical states [11] contribute to Eq. (16).

Let us now treat the case of a massive spin-2 particle. As in the massless case, we give a spinorial description of the polarization vectors, by writing the following decompositions for all the physical helicity states:

$$
\begin{aligned}
& \mathcal{E}^{\mu \nu}(k,+2)=\frac{1}{2} \bar{\sigma}^{\mu \dot{a} b} A_{b \dot{a}} \frac{1}{2} \bar{\sigma}^{\nu \dot{c} d} B_{d \dot{c}} \\
& \mathcal{E}^{\mu \nu}(k,+1)=\frac{1}{2} \bar{\sigma}^{\mu \dot{a} b} C_{b \dot{a}} \frac{1}{2} \bar{\sigma}^{\nu \dot{c} d} D_{d \dot{c}} \\
& \mathcal{E}^{\mu \nu}(k, 0)=\frac{1}{2} \bar{\sigma}^{\mu \dot{a} b} E_{b \dot{a}} \frac{1}{2} \bar{\sigma}^{\nu \dot{c} d} F_{d \dot{c}} \\
& \mathcal{E}^{\mu \nu}(k,-1)=\frac{1}{2} \bar{\sigma}^{\mu \dot{a} b} G_{b \dot{a}} \frac{1}{2} \bar{\sigma}^{\nu \dot{c} d} H_{d \dot{c}} \\
& \mathcal{E}^{\mu \nu}(k,+2)=\frac{1}{2} \bar{\sigma}^{\mu \dot{a} b} I_{b \dot{a}} \frac{1}{2} \bar{\sigma}^{\nu \dot{c} d} J_{d \dot{c}}
\end{aligned}
$$

$$
A_{a \dot{b}}=m a_{1}^{2}\left(\frac{a_{1}}{a_{2}} \varepsilon_{a \dot{b}}(k, 0)+\frac{1}{\sqrt{2}} \varepsilon_{a \dot{b}}(k,+1)-\frac{a_{1}^{2}}{a_{2}^{2}} \frac{1}{\sqrt{2}} \varepsilon_{a \dot{b}}(k,-1)\right)=\frac{a_{2}^{2}}{b_{2}^{2}} B_{a \dot{b}},
$$

where $\varepsilon_{a b}(k, \lambda)$ are the helicity spinors of a massive spin-1 particle of momentum $k$ [6], i.e.,

$$
\varepsilon_{a \dot{b}}(k,+1)=\frac{\sqrt{2}}{m} q_{a} \bar{p}_{\dot{b}}, \quad \varepsilon_{a \dot{b}}(k, 0)=\frac{1}{m}\left(p_{a} \bar{p}_{\dot{b}}-q_{a} \bar{q}_{\dot{b}}\right), \quad \varepsilon_{a \dot{b}}(k,-1)=\frac{\sqrt{2}}{m} p_{a} \bar{q}_{\dot{b}} .
$$

We obtain similar results for the remaining pairs of spinors of Eq. (17). If we take into account the normalization condition Eq. (2), we obtain the normalized helicity wave function for a massive spin-2 particle in the Weyl-van der Waerden notation, up to a phase factor

$$
\begin{aligned}
& \mathcal{E}^{\mu \nu}(k,+2)=\frac{1}{4} \bar{\sigma}^{\mu \dot{a} b} \bar{\sigma}^{\nu \dot{c} d} \frac{2}{m^{2}} \bar{p}_{\dot{a}} q_{b} \bar{p}_{\dot{c}} q_{d} \\
& \mathcal{E}^{\mu \nu}(k,+1)=\frac{1}{4} \bar{\sigma}^{\mu \dot{a} b} \bar{\sigma}^{\nu \dot{c} d} \frac{1}{m^{2}}\left[\left(\bar{p}_{\dot{a}} p_{b}-\bar{q}_{\dot{a}} q_{b}\right) \bar{p}_{\dot{c}} q_{d}+\bar{p}_{\dot{a}} q_{b}\left(\bar{p}_{\dot{c}} p_{d}-\bar{q}_{\dot{c}} q_{d}\right)\right] \\
& \mathcal{E}^{\mu \nu}(k, 0)=\frac{1}{4} \bar{\sigma}^{\mu \dot{a} b} \bar{\sigma}^{\nu \dot{c} d} \frac{2}{m^{2} \sqrt{6}}\left[\left(\bar{p}_{\dot{a}} p_{b}-\bar{q}_{\dot{a}} q_{b}\right)\left(\bar{p}_{\dot{c}} p_{d}-\bar{q}_{\dot{c}} q_{d}\right)-\bar{p}_{\dot{a}} q_{b} \bar{q}_{\dot{c}} p_{d}-\bar{q}_{\dot{a}} p_{b} \bar{p}_{\dot{c}} q_{d}\right] \\
& \mathcal{E}^{\mu \nu}(k,-1)=-\frac{1}{4} \bar{\sigma}^{\mu \dot{a} b} \bar{\sigma}^{\nu \dot{c} d} \frac{1}{m^{2}}\left[\left(\bar{p}_{\dot{a}} p_{b}-\bar{q}_{\dot{a}} q_{b}\right) \bar{q}_{\dot{c}} p_{d}+\bar{q}_{\dot{a}} p_{b}\left(\bar{p}_{\dot{c}} p_{d}-\bar{q}_{\dot{c}} q_{d}\right)\right], \\
& \mathcal{E}^{\mu \nu}(k,-2)=\frac{1}{4} \bar{\sigma}^{\mu \dot{a} b} \bar{\sigma}^{\nu \dot{c} d} \frac{2}{m^{2}} \bar{q}_{\dot{a}} p_{b} \bar{q}_{\dot{c}} p_{d} .
\end{aligned}
$$


We can verify that the sum over the polarization states, in vectorial form, is given by

$$
\begin{aligned}
& \sum_{\lambda}\left[\mathcal{E}^{\mu \nu}(k, \lambda)\right]^{\dagger} \mathcal{E}^{\alpha \beta}(k, \lambda) \\
& =\frac{1}{2}\left(\mathcal{K}^{\mu \alpha} \mathcal{K}^{\nu \beta}+\mathcal{K}^{\mu \beta} \mathcal{K}^{\nu \alpha}-\frac{2}{3} \mathcal{K}^{\mu \nu} \mathcal{K}^{\alpha \beta}\right)
\end{aligned}
$$

where

$$
\mathcal{K}^{\mu \nu}=-g^{\mu \nu}+\frac{k^{\mu} k^{\nu}}{m^{2}}
$$

Our results, Eqs. (15) and (23), can be very useful in the evaluation of invariant amplitudes involving massless and massive spin- 2 particles. In the first case, we can take advantage of the freedom in the choice of the gauge spinor $r$ to eliminate some Feynman diagrams contributing to a given process. In the massive case, we can make convenient choices of the momenta $p$ and $q$, defined in Eq. (18), to reduce the number of contributions.

This work was partially supported by Conselho Nacional de Desenvolvimento Científico e Tecnológico, CNPq (Brazil).
[1] P. de Causmaecker, R. Gastmans, W. Troost, and T.T. Wu, Phys. Lett. 105B, 215 (1981); J.F. Gunion and Z. Kunszt, ibid. 161B, 333 (1985); R. Kleiss and W.J. Stirling, Nucl. Phys. B262, 235 (1985); Z. Xu, D.-H. Zhang, and L. Chang, ibid. B291, 392 (1987).

[2] G.R. Farrar and F. Neri, Phys. Lett. 130B, 109 (1983); A. Kersch and F. Scheck, Nucl. Phys. B263, 475 (1986); K. Hagiwara and D. Zeppenfeld, ibid. B274, 1 (1986).

[3] F.A. Berends and W.T. Giele, Nucl. Phys. B294, 700 (1987).

[4] H. Weyl, The Theory of Groups and Quantum Mechanics (Methuen, London, 1931); Z. Phys. 56, 330 (1929); B.L. van der Waerden, Gött. Nachr. K1, 100 (1929); E.M. Corson, Introduction to Tensors, Spinors, and Relativistic Wave-Equations (Hafner, New York, 1953).

[5] F.A. Berends and W.T. Giele, Nucl. Phys. B306, 759 (1988); K. Hagiwara and D. Zeppenfeld, ibid. B313, 560 (1989); F.A. Berends, W.T. Giele, and H. Kuijf, ibid.
B321, 39 (1989); B333, 120 (1990).

[6] S.F. Novaes and D. Spehler, Nucl. Phys. (to be published).

[7] J. Wess and J. Bagger, Supersymmetry and Supergravity (Princeton University Press, Princeton, 1983).

[8] M.J.G. Veltman, in Quantum Theory of Gravitation, Les Houches, France, 1975, edited by R. Balian and J. Zinn-Justin, Les Houches Summer School Proceedings Vol. XXIII (North-Holland, Amsterdam, 1975).

[9] S. Weinberg, Gravitation and Cosmology: Principles and Applications of the General Theory of Relativity (Wiley, New York, 1972).

[10] F.A. Berends, W.T. Giele, and H. Kuijf, Phys. Lett. B 211, 91 (1988); J.G.M. Kuijf, Ph.D. thesis, Leiden University, 1990.

[11] H. Van Dam and M. Veltman, Nucl. Phys. B22, 397 (1970). 